\title{
Atomic structures of Tsai-type icosahedral quasicrystals and approximants
}

\author{
T. Yamada \\ Faculty of Science, Department of Applied Physics, Tokyo University of Science, Tokyo, 125-8585, Japan \\ tsunetomo.yamada@rs.tus.ac.jp
}

The first stable binary icosahedral quasicrystals (iQCs) were found in $\mathrm{Cd}-\mathrm{Yb}$ and $\mathrm{Cd}-\mathrm{Ca}$ alloy systems [1, 2], which was followed by finding of nine isostructural iQCs in Cd- $R(R=\mathrm{Y}, \mathrm{Gd}-\mathrm{Tm})$ [3] and $\mathrm{Zn}-\mathrm{Sc}$ systems [4]. The structural type of these iQC is called Tsaitype, and it has been extended to ternary or quaternary alloys by atomic substitutions.

Higher-dimensional structure analysis of the $\mathrm{Cd}-\mathrm{Yb}$ iQC by single-crystal $\mathrm{X}$-ray diffraction revealed that the atomic structure consists of two main building blocks, rhombic triacontahedron (RT) and acute rhombohedron (AR) units [5]. In ternary iQCs, the structure analysis becomes more difficult because occupational disorder has to be taken into account in the 6D structure model. Furthermore, recent studies have shown that some sites are preferentialy occupied by the substituting elements [6,7], which indicates that the higher-dimensional structure model must be optimized to ternary iQCs. To build such model, knowledge of atomic structures in ternary quasicrystal approximants (APs) is quite important.

In the first part of my talk, I will present the superstructure and basic structure of ternary $\mathrm{Yb}-\mathrm{Cd}-\mathrm{Mg} 1 / 1 \mathrm{APs}$ with the compositions $\mathrm{Yb}_{12.9} \mathrm{Cd}_{78.4} \mathrm{Mg}_{8.8}$ and $\mathrm{Yb}_{13.3} \mathrm{Cd}_{64.2} \mathrm{Mg}_{22.5}$ [8]. The former was determined to have a face-centred packing structure comprising two distinguishable RT units (space group $F d 3, a=31.377(1) \AA$ ), while the latter was found to have a body-centred packing structure made of identical RT units (space group $\operatorname{Im} 3, \mathrm{a}=15.7596(4) \AA$ ). The distinction between the two types of RT units in the superstructure is based on the positional disorder of the first tetrahedron shell and the relative $\mathrm{Cd} / \mathrm{Mg}$ occupancy at sites (48h) in the fourth icosidodecahedron shell.

In the second part, I will introduce a Python package (PyQCstrc) for building the higher-dimensional models of iQCs [9] and present a modification of six-dimensional structural model for the primitive Tsai-type iQCs so as to incorporate the selective $\mathrm{Cd} / \mathrm{Mg}$ occupation found in the Cd-Mg-Yb 1/1 APs [7].

[1] A.P. Tsai, J.Q. Guo, E. Abe, H. Takakura, and T.J. Sato, (2000), Nature, 408, 537-538.

[2] Guo, J. Q., Abe, E., Tsai, A. P. Phys. Rev. B. (2000), 62, R14605-R14608.

[3] Goldman, A. I., Kong, T., Kreyssig, A., Jesche, A., Ramazanoglu, M., Dennis, K. W., Bud'ko, S. L., Canfield, P. C., (2013), Nat. Mater, 12, 714 -718 .

[4] Canfield, P. C., Caudle, M. L., Ho, C. S., Kreyssig, A., Nandi, S., Kim, M. G., ... \& Goldman, A. I. (2010), Phys. Rev. B, 81(2), 020201.

[5] Takakura, H., Pay Gómez, C., Yamamoto, A., de Boissieu, M., and Tsai, A.P., (2007), Nat. Mater. 6, 58-63.

[6] Pay Gómez, C. \& Tsai, A. P. (2013). Comptes Rendus Physique, 15(1), 1-10.

[7] Yamada, T., Takakura, H., de Boissieu, M. and Tsai, A.-P., (2017), Acta Cryst. B73, 1125-1141.

[8] Yamada, T, (2021), Phil. Mag., 101(3), 257-275.

[9] Yamada, T, J. Appl. Cryst., in press

\section{Keywords: Quasicrystal, Approximant}

This work was supported by JSPS KAKENHI grants (numbers JP18K13987, JP19H05818). 